\title{
Nonsplit Geodetic Number of a Lict Graph
}

\author{
Venkanagouda M Goudar \\ Department of Mathematics, \\ Sri Siddhartha Institute of \\ Technology, \\ Tumkur, Karnataka, India
}

\author{
Tejaswini K.M. \\ Research Scholar, \\ Sri Gautama Research \\ Center(Affiliated to Kuvempu \\ University) \\ Tumkur, Karnataka, India
}

\author{
Venkatesha, \\ Department of Mathematics, \\ Kuvempu University, \\ Shankarghatta, Shimoga, \\ Karnataka, India
}

\begin{abstract}
A set $S \subseteq V(\eta(G))$ is a non split geodetic set of $\eta(G)$, if S is a geodetic set and $\langle V-S\rangle$ is connected. The nonsplit geodetic number of a lict graph $\eta(G)$, denoted by $g_{n s}(\eta(G))$ , is the minimum cardinality of a nonsplit geodetic set of $\eta(G)$. The bounds on non split geodetic number in terms of elements of $\mathrm{G}$ and covering number of $\mathrm{G}$. Further the relationship between nonsplit geodetic number and geodetic number of a graph is established.
\end{abstract}

\section{General Terms}

AMS Mathematics Subject Classification (2010): 05C05, $05 \mathrm{C} 12$.

\section{Keywords}

Cartesian product, Distance, Edge covering number, geodetic number, Vertex covering number.

\section{INTRODUCTION}

In this paper we follow the notations and undefined terms of [3]. As usual $n=|V|$ and $m=|E|$ denote the number of vertices and edges of a graph $G$ respectively. The graphs considered here are finite, undirected and simple and have at least one component which is not complete or at least two non trivial components. We refer [3] for unexplained terminology and notation. For any graph $\mathrm{G}=(\mathrm{V}, \mathrm{E})$, the Lict graph $\eta(G)$ [4], whose vertex set is the union of the set of edges and the set of cut vertices of $\mathrm{G}$ in which two vertices of $\eta(G)$ adjacent if and only if the corresponding edges of $G$ are adjacent or the corresponding members of $\mathrm{G}$ are incident. The distance $\mathrm{d}(\mathrm{u}$, $v)$ between two vertices $u$ and $v$ in a connected graph $G$ is the length of a shortest $\mathrm{u}-\mathrm{v}$ path in G. It is well known that this distance is a metric on the vertex set V (G). For a vertex v of $\mathrm{G}$, the eccentricity $\mathrm{e}(\mathrm{V})$ is the distance between $\mathrm{v}$ and a vertex farthest from $v$. The minimum eccentricity among the vertices of $\mathrm{G}$ is radius, rad $\mathrm{G}$ and the maximum eccentricity is the diameter, diam $\mathrm{G}$.

A $u-v$ path of length $d(u, v)$ is called a $u-v$ geodesic. We define $I[u, v]$ to the set (interval) of all vertices lying on some $\mathrm{u}-\mathrm{v}$ geodesic of $\mathrm{G}$ and for a nonempty subset $\mathrm{S}$ of $\mathrm{V}(\mathrm{G})$, $I[S]=U_{u, v \in S} I(u, v)$.

A set $\mathrm{S}$ of vertices of $\mathrm{G}$ is called a geodetic set in $\mathrm{G}$ if $\mathrm{I}[\mathrm{S}]=$ $\mathrm{V}(\mathrm{G})$ and a geodetic set of minimum cardinality is a minimum geodetic set. The cardinality of a minimum geodetic set in $\mathrm{G}$ is called the geodetic number $\mathrm{g}(\mathrm{G})$.
Nonsplit geodetic number of a graph was studied in [6]. A geodetic set $\mathrm{S}$ of a graph $\mathrm{G}=(\mathrm{V}, \mathrm{E})$ is a nonsplit geodetic set if the induced subgraph $<\mathrm{V}-\mathrm{S}>$ is connected. The nonsplit geodetic number $g_{n s}(G)$ is the minimum cardinality of a nonsplit geodetic set. Geodetic number of a lict graph was studied in [6]. Geodetic number of a lict graph $\eta(G)$ is a set $S^{\prime}$ of vertices $\eta(G)=\mathrm{H}$ is called a geodetic set in $\mathrm{H}$ if $I\left[S^{\prime}\right]$ $=\mathrm{V}(\mathrm{H})$, and a geodetic set of minimum cardinality is a Lict geodetic number of $\mathrm{G}$ and is denoted by $g(\eta(G))$. Now we define nonsplit geodetic number of a lict graph. A set $S^{\prime}$ of vertices of $\eta(G)=\mathrm{H}$ is called the nonsplit geodetic set in $\mathrm{H}$ if the induced subgraph $V(H)-S^{\prime}$ is connected and a nonsplit geodetic set of minimum cardinality is the nonsplit geodetic number of $\eta(G)$ and is denoted by $g_{n s}(\eta(G))$.

A vertex $v$ is an extreme vertex in a graph $\mathrm{G}$, if the subgraph induced by its neighbors is complete. A vertex cover in a graph $\mathrm{G}$ is a set of vertices that covers all edges of $\mathrm{G}$. The minimum number of vertices in a vertex cover of $\mathrm{G}$ is the vertex covering number $\alpha_{0}(G)$ of $\mathrm{G}$. An edge cover of a graph $\mathrm{G}$ without isolated vertices is a set of edges of $\mathrm{G}$ that covers all the vertices of $\mathrm{G}$. The edge covering number $\alpha_{1}(G)$ of a graph $\mathrm{G}$ is the minimum cardinality of an edge cover of $\mathrm{G}$

\section{PRELIMINARY RESULTS}

Theorem 2.1[1] Every geodetic set of a graph contains its extreme vertices.

Theorem 2.2 [1] For any tree $\mathrm{T}$ with $\mathrm{k}$ end edges, $\mathrm{g}(\mathrm{T})=$ $\mathrm{k}$.

Theorem 2.3[3] For any graph $\mathrm{G}$ of order $\mathrm{n}$, $\alpha_{1}(G)+\beta_{1}(G)=n$.

Theorem 2.4[1] If $\mathrm{G}$ is a nontrivial connected graph, then $g(G) \leq g\left(G \times K_{2}\right)$

Theorem 2.5[3] For any path $P_{n}$, the edge covering number is $\alpha_{1}\left(P_{n}\right)=\left\{\begin{array}{cl}\frac{n}{2} & \text { if niseven } \\ \frac{n+1}{2} & \text { if nisodd }\end{array}\right.$ 
Theorem 2.6[3] For any path $P_{n}$, the vertex covering number is $\alpha_{0}\left(P_{n}\right)=\left\{\begin{array}{cl}\frac{n}{2} & \text { if niseven } \\ \frac{n-1}{2} & \text { if nisodd }\end{array}\right.$

Theorem 2.7[5] For any tree T, $g(\eta(T))=n$.

Theorem 2.8 For any tree T, $\eta(T)$ is a Block graph.

Theorem 2.9 For any graph $g(\eta(G)) \leq g_{n s}(\eta(G))$

\section{MAIN RESULTS}

Theorem 3.1 For any tree of order $\mathrm{n}, g_{n s}[\eta(T)]=n$, where $\mathrm{n}$ be the number of vertices of $\mathrm{T}$.

Proof. Let $\mathrm{S}$ be the set of all extreme vertices of a lict graph of a tree. By the Theorem 2.1, we obtain $g[\eta(T)] \geq S$. This implies $g[\eta(T)] \geq S$. On the other hand for an internal vertex $\mathrm{v}$ of $\mathrm{T}$ there exists end vertices $\mathrm{x}, \mathrm{y}$ of $\mathrm{T}$, such that $v \in I(S)$ and $I(S)=V(T)$. Thus, $\quad g_{n s}[\eta(T)] \leq S$. Therefore $g_{n s}[\eta(T)]=S$. Also, every geodetic set of $S^{\prime}$ of T must contain $\mathrm{S}$, is the minimum geodetic set and $V-S$ is connected. Thus $\mathrm{S}$ itself a minimum nonsplit geodetic set of $\mathrm{G}=\eta(T)$ i.e $g_{n s}[\eta(T)]=S$. Now by the definition, the pendant edges and cut vertices of a tree are the extreme vertices of a lict graph. Since the number of vertices in $\mathrm{T}$ is the sum of cut vertices and the number of pendant edges. Hence the minimum nonsplit geodetic set is the number of vertices in tree T. Thus $g_{n s}[\eta(T)]=n$. Hence the proof.

Theorem 3.2 For any path $P_{n}$ of order n, $g_{n s}\left[\eta\left(P_{n}\right)\right]=n$ where $\mathrm{n}$ is the number of vertices.

Proof . Proof follows from the above Theorem.

Theorem 3.3 For any path $P_{n}$ of order n, $g_{n s}\left[\eta\left(P_{n}\right)\right]=\alpha_{1}\left(P_{n}\right)+\beta_{1}\left(P_{n}\right)$.

Proof. Since $\alpha_{1}\left(P_{n}\right)+\beta_{1}\left(P_{n}\right)=n$ and also $g_{n s}\left[\eta\left(P_{n}\right)\right]=n$ ,we have $g_{n s}\left[\eta\left(P_{n}\right)\right]=\alpha_{1}\left(P_{n}\right)+\beta_{1}\left(P_{n}\right)$. Hence the proof.

Theorem 3.4 For any path $P_{n}$ of order n, $g_{n s}\left[\eta\left(P_{n}\right)\right]=\omega\left(P_{n}\right)+c_{i}$ where $\omega\left(P_{n}\right)$ and $c_{i}$ are the clique and cut vertices of $P_{n}$ respectively.

Proof. Let $S=\left\{u_{1}, u_{2}, u_{3}, \ldots, u_{k}\right\}$ be the geodetic set of $\eta\left(P_{n}\right)$. Now, consider any set $S^{\prime}=S-\left\{u_{i}\right\}$, with extreme vertex $u_{i} \in S$. Let us consider any vertices $u_{j}, u_{k}$ of $S^{\prime}$. Since each block in $\eta\left(P_{n}\right)$ is $K_{3}, u_{i}$ does not lies in $u_{j}-u_{k}$ geodesic. So $S^{\prime}$ is not a geodetic set of $\eta\left(P_{n}\right)$.
Hence $\mathrm{S}$ is a minimum nonsplit geodetic set, further $V-S$ is connected. So $|S|=n=\omega\left(P_{n}\right)+c_{i}$. Hence the proof.

Theorem 3.5 For any path $P_{n}$ of order n, $g_{n s}\left[\eta\left(P_{n}\right)\right]=\left\{\begin{array}{cc}2 \alpha_{0}\left(P_{n}\right), & n \text { is even } \\ 2 \alpha_{0}\left(P_{n}\right)+1, & n \text { is odd }\end{array}\right.$

Proof. Let $P_{n}$ be the path with $n \geq 4$ vertices. Consider $V=\left\{v_{1}, v_{2}, v_{3}, \ldots, v_{n}\right\} \quad$ be the vertices and $E=\left\{\left(v_{i}, v_{i+1}\right)\right\}, i=1,2,3, \ldots$ be the edge set of path $P_{n}$. By the Theorem 2.6, the vertex covering number is a minimum cardinality of vertex cover of $P_{n}$. We have the following cases,

Case(i). Suppose $n$ is even, by the Theorem 2.6 we have

$$
\begin{aligned}
& \alpha_{0}\left(P_{n}\right)=\frac{n}{2} \\
\Rightarrow \quad & n=2 \alpha_{0}\left(P_{n}\right)
\end{aligned}
$$

Since $g_{n s}\left[\eta\left(P_{n}\right)\right]=n$, we have $g_{n s}\left[\eta\left(P_{n}\right)\right]=2 \alpha_{0}\left(P_{n}\right)$.

Case(ii). Suppose $\mathrm{n}$ is odd , by the Theorem 2.6 we have

$$
\begin{aligned}
& \alpha_{0}\left(P_{n}\right)=\frac{n-1}{2} \\
\Rightarrow & n=2 \alpha_{0}\left(P_{n}\right)+1
\end{aligned}
$$

Since $g_{n s}\left[\eta\left(P_{n}\right)\right]=n$, we have $g_{n s}\left[\eta\left(P_{n}\right)\right]=2 \alpha_{0}\left(P_{n}\right)+1$.

Hence the proof.

Theorem 3.6 For any path $P_{n}$ of order n, $g_{n s}\left[\eta\left(P_{n}\right)\right]=\left\{\begin{array}{cc}2 \alpha_{1}\left(P_{n}\right), & n \text { is even } \\ 2 \alpha_{1}\left(P_{n}\right)-1, & n \text { is odd }\end{array}\right.$

Proof. Let $P_{n}$ be the path with $n \geq 4$ vertices. Consider $V=\left\{v_{1}, v_{2}, v_{3}, \ldots, v_{n}\right\} \quad$ be the vertices and $E=\left\{\left(v_{i}, v_{i+1}\right)\right\}, i=1,2,3, \ldots$ be the edge set of path $P_{n}$. By the Theorem 2.5 the edge covering number is a minimum cardinality of edge cover of $P_{n}$. We have the following cases

Case(i). Suppose $\mathrm{n}$ is even, by the Theorem 2.5 , we have

$$
\begin{aligned}
& \alpha_{1}\left(P_{n}\right)=\frac{n}{2} \\
\Rightarrow \quad & n=2 \alpha_{1}\left(P_{n}\right)
\end{aligned}
$$

Since $g_{n s}\left[\eta\left(P_{n}\right)\right]=n$, we have $g_{n s}\left[\eta\left(P_{n}\right)\right]=2 \alpha_{1}\left(P_{n}\right)$

Case(ii). Suppose $\mathrm{n}$ is odd, by the Theorem 2.5, we have

$$
\begin{aligned}
& \alpha_{1}\left(P_{n}\right)=\frac{n+1}{2} \\
\Rightarrow & n+1=2 \alpha_{1}\left(P_{n}\right) \\
\Rightarrow & n=2 \alpha_{1}\left(P_{n}\right)-1
\end{aligned}
$$


Since $g_{n s}\left[\eta\left(P_{n}\right)\right]=n$, we have $g_{n s}\left[\eta\left(P_{n}\right)\right]=2 \alpha_{1}\left(P_{n}\right)-1$.

Hence the proof

\section{ADDING AN END EDGE}

For an edge $e=(u, v)$ of a graph $G$ with $\operatorname{deg}(u)=1$ and $\operatorname{deg}(v)>1$, we call e an end-edge and $u$ an end-vertex.

Theorem 4.1 If $G^{\prime}$ is a graph obtained by adding a pendant vertex to $G=C_{n}$ then $g_{n s}\left[\eta\left(G^{\prime}\right)\right]=k+3$, k be the number of pendant vertices.

Proof: Let $\left\{e_{1}, e_{2}, \ldots, e_{n}, e_{1}\right\}$ be a cycle with $\mathrm{n}$ vertices which is even and $G^{\prime}$ be the graph obtained from $\mathrm{G}=C_{n}$ by adding end edges $\left\{\mathrm{u} \mathrm{v}_{\mathrm{i}}\right\}, \mathrm{i}=1,2, \ldots, \mathrm{k}$ such that $u \in G$ and $v_{i} \notin G$. By the definition of lict graph $\eta\left(G^{\prime}\right)$ has $\left\langle K_{k+3}\right\rangle$ as an induced subgraph. Also the edges $\left\{\mathrm{u} \mathrm{v}_{\mathrm{i}}\right\}, \mathrm{i}=1,2, \ldots, \mathrm{k}$ and the cutvertex $\mathrm{u}$ becomes the vertices of $\eta\left(G^{\prime}\right)$. These belongs to some geodetic set of $\eta\left(G^{\prime}\right)$. Hence $\left\{u, e_{1}, e_{2}, \ldots, e_{k}, e_{l}, e_{m}\right\}$ are the vertices of $\eta\left(G^{\prime}\right)$ where $e_{l}, e_{m}$ are the edges incident on the antipodal vertex of $\mathrm{u}$ in $G^{\prime}$ and these vertices belongs to some geodetic set of $\eta\left(G^{\prime}\right)$. Thus $\eta\left(G^{\prime}\right)=C_{n} U K_{k+3}$. Let $S=\left\{u, e_{1}, e_{2}, \ldots e_{k}, e_{l}, e_{m}\right\}$ be the geodetic set. Suppose $S^{\prime}=\left\{u, e_{1}, e_{2}, \ldots e_{k}\right\}$ be the set vertices $\eta\left(G^{\prime}\right)$ such that $\left|S^{\prime}\right|<|S|$ then $S^{\prime}$ is not a geodetic set of $\eta\left(G^{\prime}\right)$. Thus, $S$ is the minimum geodetic set and V-S is connected. Hence $\mathrm{S}$ is the minimum nonsplit geodetic set, therefore $g_{n s}\left[\eta\left(G^{\prime}\right)\right]=k+3$ . Hence the proof.

Theorem 4.2 If $G^{\prime}$ is a graph obtained by adding a pendant vertex to $G=C_{n}$ then $g_{n s}\left[\eta\left(G^{\prime}\right)\right]=k+2$, k be the number of pendant vertices.

Proof: Let $\left\{e_{1}, e_{2}, \ldots, e_{n}, e_{1}\right\}$ be a cycle with $\mathrm{n}$ vertices which is odd and $G^{\prime}$ be the graph obtained from $\mathrm{G}=\mathrm{Cn}$ by adding pendant edges $\{\mathrm{u} \mathrm{vi}\}, \mathrm{i}=1,2, \ldots, \mathrm{k}$ such that $u \in G, v_{i} \notin G$. By the definition of lict graph $\eta\left(G^{\prime}\right)$ has $\left\langle K_{k+2}\right\rangle$ as an induced sub graph, also the edges $\left\{\mathrm{u} \mathrm{v}_{\mathrm{i}}\right\}, \mathrm{i}=1,2, \ldots, \mathrm{k}$ becomes vertices of $\eta\left(G^{\prime}\right)$. Further the cutvertex $\mathrm{u}$ is also a vertex of $\eta\left(G^{\prime}\right)$ and it belongs to some geodetic set of $\eta\left(G^{\prime}\right)$. Hence $S=\left\{u, e_{1}, e_{2}, \ldots, e_{k}, e_{l}, e_{m}\right\}$ are the geodetic set of $\eta\left(G^{\prime}\right)$ where $e_{l}, e_{m}$ are the edges on the vertex of $\mathrm{u}$ in $G^{\prime}$. Let $e_{i}=\{a, b\} \in G$ such that $d(u, a)=d(u, b)$, in the $\eta\left(G^{\prime}\right)$, two element subsets of $\eta\left(G^{\prime}\right)$ has the property that $I(S)=V\left[\eta\left(G^{\prime}\right)\right]$. Since V-S is connected, thus $S$ itself a minimum nonsplit geodetic set of $\eta\left(G^{\prime}\right)$. Therefore $g_{n s}\left[\eta\left(G^{\prime}\right)\right]=k+2$.

Hence the proof.

\section{CARTESIAN PRODUCT OF GRAPHS}

The Cartesian product of two graphs G, $\mathrm{H}$ by $G \times H$, and it is the graph with the vertex set $V(G) \times V(H)$ specified by putting (u, v) adjacent to $\left(u^{\prime}, v^{\prime}\right)$ if and only if (1) $\mathrm{u}=u^{\prime}$ and $v v^{\prime} \in E(H)$ or (2) $\mathrm{v}=v^{\prime}$ and $u u^{\prime} \in E(G)$. A vertex $\mathrm{v}$ is an extreme vertex in a graph $\mathrm{G}$, and the subgraph induced by its neighbors is complete. A vertex cover in a graph $\mathrm{G}$ is a set of vertices that covers all edges of $\mathrm{G}$.

Theorem 5.1 For any graph $\mathrm{G}, g_{n s}\left[K_{3} \times G\right]=n$.

Proof. Consider $G=\eta\left(P_{n}\right)$. Let $K_{3} \times G$ be the graph formed from two copies $G_{1}$ and $G_{2}$ of $\mathrm{G}$ and $\mathrm{S}$ be a minimum nonsplit geodetic set of $K_{3} \times G$. Now, we define $S^{\prime}$ to be the union of those vertices of $\mathrm{S}$ in $G_{1}$ and the vertices of $G_{1}$ corresponding to vertices of $G_{2}$ belonging to S. Let $v \in V\left(G_{1}\right)$ lies on some $x-y$ geodesic where $x, y \in S$. Since $\mathrm{S}$ is a nonsplit geodetic set, by the theorem we have $g_{n s}[G]=n$ at least one of $x$ and $y$ belong to $V_{1}$. If both $x, y \in V_{1}$ then $x, y \in S^{\prime}$. Hence we may assume that $x \in V_{1}$ and $y \in V_{2}$. If $y$ corresponds to $x$ then $v=x \in S^{\prime}$. Hence, we may assume that $\mathrm{y}$ corresponds to $y^{\prime} \in S^{\prime}$ where $y \neq x$. Since $d(x, y)=d\left(x, y^{\prime}\right)+1$ and the vertex $\mathrm{v}$ lies on an $x-y$ geodesic in $K_{3} \times G$. Hence v lies on an $x-y$ geodesic in $\mathrm{G}$ that is $g(G) \leq g_{n s}\left(K_{3} \times G\right)$.

Further, let $\mathrm{S}$ contains a vertex $\mathrm{x}$ with the property that every vertex of $G_{1}$ lies on an $x-w$ geodesic in $G_{1}$ for some $w \in S$. Let $S^{\prime}$ consists of $\mathrm{x}$ together with those vertices of $G_{2}$ corresponding to those vertices in $S-\{x\}$. Thus $\left|S^{\prime}\right|=|S|$. We show that $S^{\prime}$ is a nonsplit geodetic set of $K_{3} \times G$. Hence $g_{n s}\left(K_{3} \times G\right) \leq g(G)$. Therefore $g_{n s}\left(K_{3} \times G\right)=g(G)=n$.

Hence the proof.

Theorem 5.2 For any path $P_{n}$, $g_{n s}\left[K_{3} \times \eta\left(P_{n}\right)\right]=\left\{\begin{array}{cc}2 \alpha_{0}\left(P_{n}\right), & n \text { is even } \\ 2 \alpha_{0}\left(P_{n}\right)+1, & n \text { is odd }\end{array}\right.$

Proof. Let $\alpha_{0}\left(P_{n}\right)$ be a vertex covering of a graph is a minimum cardinality of an vertex cover of a path $P_{n}$. We have the following cases,

Case(i). Suppose $\mathrm{n}$ is even, by the Theorem 2.6, we have

$$
\begin{aligned}
& \alpha_{0}\left(P_{n}\right)=\frac{n}{2} \\
& \Rightarrow n=2 \alpha_{0}\left(P_{n}\right)
\end{aligned}
$$

Since $g_{n s}\left[K_{3} \times \eta\left(P_{n}\right)\right]=n$, we have $g_{n s}\left[K_{3} \times \eta\left(P_{n}\right)\right]=2 \alpha_{0}\left(P_{n}\right)$.

Case(ii). Suppose $n$ is odd, by the Theorem 2.6, we have

$$
\alpha_{0}\left(P_{n}\right)=\frac{n-1}{2}
$$




$$
\Rightarrow \quad n=2 \alpha_{0}\left(P_{n}\right)+1
$$

Since $g_{n s}\left[K_{3} \times \eta\left(P_{n}\right)\right]=n$, we have $g_{n s}\left[K_{3} \times \eta\left(P_{n}\right)\right]=2 \alpha_{0}\left(P_{n}\right)+1$.

Hence the proof.

\section{CONCLUSION}

In this paper we studied the nonsplit geodetic number of lict graph of a graph and obtain some results on the Cartesian product of a graph. Further, the results on adding an end edge to a cycle $C_{n}$ and its lict graph characterised.

\section{REFERENCES}

[1] G. Chartrand, F. Harary, and P.Zhang, On the geodetic number of a graph.Networks.39, 1-6 (2002)
[2] G. Chartrand and P.Zhang, Introduction to Graph Theory, Tata McGraw Hill Pub.Co.Ltd.(2006).

[3] F.Harary, Graph Theory, Addison-Wesely, Reading, MA,(1969)

[4] V.R.Kulli and M.H. Muddebihal. Lict Graph and Litact Graph of a Graph, Journal of Analysis and Computation, Vol. 2.No. 133-43.(2006).

[5] Tejaswini K.M, Venkanagouda M Goudar, Venkatesha \& M.H. Muddebihal, "ON THE LICT GEODETIC NUMBER OF A GRAPH", International Journal of Mathematics and Computer Applications Research Vol.2, Issue 3 65-69, (2012).

[6] Venkanagouda.M.Goudar,Tejaswini K.M.,Venkatesha, Nonsplit Geodetic Number of a Graph, Indian Journal of pure and applied mathematics(submitted). 